\title{
Design of a Transverse Flux Linear Motor
}

\author{
Junghwan Chang ${ }^{1 *}$, Jiwon Kim², and Dohyun Kang² \\ ${ }^{1}$ Department of Electrical Engineering, Dong-A University, Busan 604-714, Korea \\ ${ }^{2}$ Electric Motor Research Center, KERI, Changwon City 641-120, Korea
}

(Received 29 November 2010, Received in final form 27 January 2011, Accepted 16 February 2011)

\begin{abstract}
This paper presents design procedures of a transverse flux linear motor (TFLM). The minimum and maximum flux linkage was determined by the simplified equivalent magnetic circuit and estimated average magnetic flux density at the air gap region by considering the shape of applied magnetomotive force (MMF). With this information, the number of turns of each phase winding was calculated based on the amplitude of applied voltage and speed of a mover. The rated current, coil diameter, and winding area were obtained with the aid of an empirical formula for determining the required MMF. The usefulness of the proposed design method for TFLM is verified by the three-dimensional equivalent magnetic circuit network (EMCN) method and the experimental results of prototyped machine.
\end{abstract}

Keywords : equivalent magnetic circuit network, flux concentration, linear motor, transverse flux

\section{Introduction}

A transverse flux linear motor (TFLM) with a permanent magnet excitation is devised by combining high-energy permanent magnets and magnetic circuit designs for flux concentration. Among other things, the most important feature of a TFLM is that there is no competition between electric and magnetic loading. This increases the design flexibility of the TFLM when compared with other longitudinal flux machines. However, in spite of this advantage, three-dimensional flux flows in a TFLM creates a complex core design and assembly and makes two-dimensional numerical analysis of the motor difficult. Therefore, three-dimensional finite element analysis is required. However, this type of calculation requires long simulation times, making them unsuitable for an initial design $[1,2]$.

This paper presents design procedures for a TFLM. The ratios of the important design variables are determined by considering thrust density and detent force, with the help of previous studies of TFLM [3]. The simplified equivalent magnetic circuit and estimated average magnetic flux densities at the air gap region are used to calculate the minimum and maximum flux linkages with armature winding. These two values determine the number of turns of

*Corresponding author: Tel: +82-51-200-7735

Fax: +82-51-200-7743, e-mail: cjhwan@dau.ac.kr the winding by considering the amplitude of the applied voltage and rated speed. Finally, with the help of an empirical formula for determining the required magnetomotive force (MMF), the rated current, coil diameter, and winding area could be obtained. With this proposed design method, a parametrical study can be performed to ascertain the main influencing parameters in motor performances without the typical long simulation times required by three-dimensional finite element analysis. Fig. 1 shows the entire process of the design flow of the TFLM. The usefulness of the proposed design method is verified by a

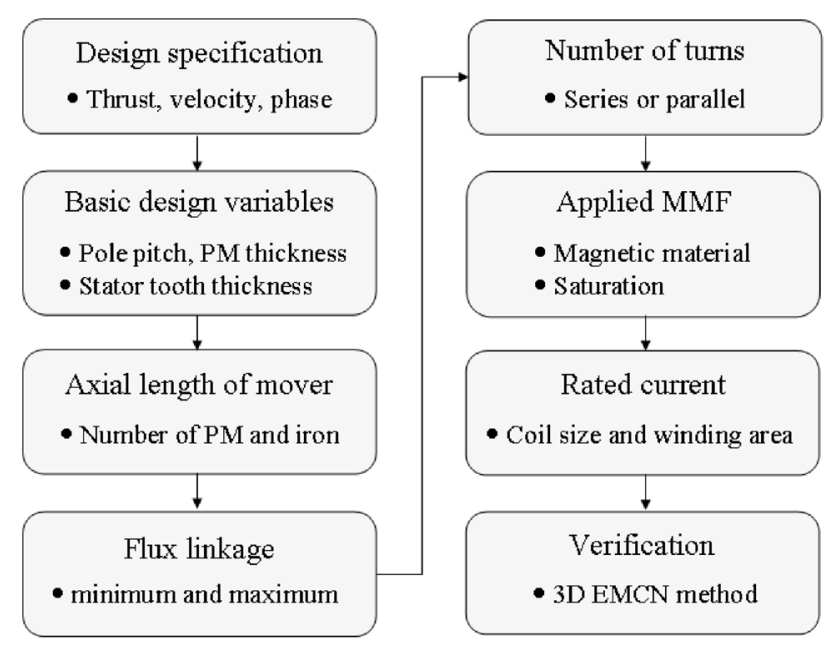

Fig. 1. Design flow of TFLM. 


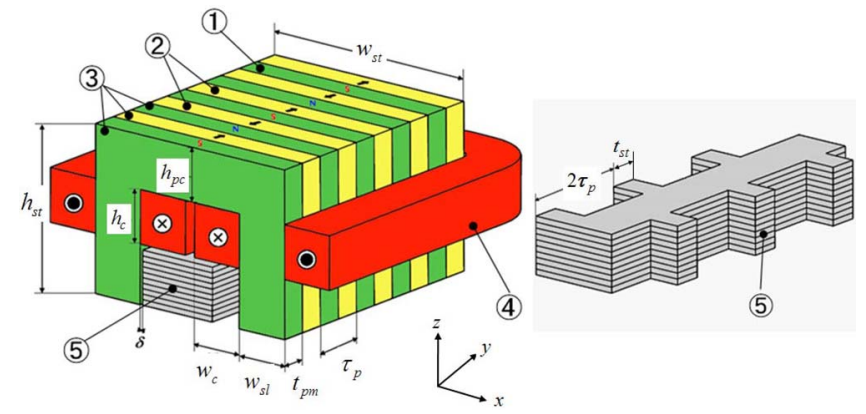

Fig. 2. TFLM structure ( (1) soft magnetic composite material; (2) permanent magnet; (3) mover; (4) coil; (5) stator).

three-dimensional equivalent magnetic circuit network (EMCN) method and the experimental results of the prototyped machine.

\section{Design of TFLM}

\subsection{Transverse flux linear motor}

Fig. 2 shows a type of TFLM. Due to the three-dimensional flux flow, it is difficult to adapt conventional laminated steel assemblies to the entire TFLM structure. In this design, the stator consists of a plurality of laminated cores in the z-direction. In the case of the mover, however, each core should be laminated from the inside to the outside of the mover to reduce eddy current loss. Instead of this complicated mover structure, soft magnetic composite (SMC) material is employed for the mover core. Even though it possesses a low saturation magnetic flux density, it allows three-dimensional flux flow and is manufactured with relative ease. One distinguishing feature of this design is that the mover has all active parts, such as permanent magnet and windings, and the long stator has only passive iron parts to complete the magnetic circuit. With this advantage, the structure for eliminating unwanted attractive forces between the stator and mover makes this design allows use in direct drive applications with relatively long strokes.

\subsection{Basic design of TFLM}

\subsubsection{Design specification}

In designing a machine, One must determine the basic design specifications at first. This includes the required thrust, number of phase, and rated speed. Because a TFLM has many variants, its basic structure should also be determined in the initial design stages through consideration of its final, intended application.

\subsubsection{Basic design variables}

In designing a TFLM, the geometrical dimensions related to magnetic loading can be selected independent of those related to electric loading. For example, the axial length of the pole pitch and permanent magnet setting the magnetic loading can be determined without competing with the lateral width of the motor setting of the electric loading. In a previous study of a TFLM [3], to obtain the maximum thrust density, the pole pitch $\left(\tau_{p}\right)$, stator tooth thickness $\left(t_{s t}\right)$, and permanent magnet thickness $\left(t_{p m}\right)$ have to satisfy the following ratio:

$$
\begin{aligned}
& t_{s t} / \tau_{p} \approx 0.7 \\
& t_{p m} / \tau_{p} \approx 0.5
\end{aligned}
$$

Although decreasing the pole pitch can increase thrust density, the high level of leakage flux and speed of the mover set practical limitations on the length of the pole pitch. In some cases, minimization of the detent force is the main consideration, with the abovementioned ratios between the parameters capable of being changed with some sacrifice of thrust.

\subsubsection{Axial length of the mover}

The axial length of each phase of a mover can be determined by thrust density $\left(F_{x d}\right)$ and available space for each application:

$$
\text { Axial length of mover }=\frac{\text { thrust } / \text { phase }}{F_{x d} \cdot \text { force generating height }}
$$

With the determined value of $\tau_{p}$ and axial length of a mover, the total number of pieces of the permanent magnets and SMC cores can be obtained. For manufacturing convenience, both ends of the mover were covered with SMC cores.

\subsubsection{Flux linkage}

To determine the number of turns of each phase winding, the maximum time variation of the flux linkage is calculated. Under no-load conditions, the flux linkage has a minimum value when the mover is located at the stable equilibrium point. After the half pole pitch from that point, the flux linkage has a maximum value by considering the sinusoidal shape of the applied MMF. Fig. 3(a) shows the magnetic flux flow of the TFLM at the minimum flux linkage condition. The magnetic flux from the permanent magnet is concentrated into a single section of the SMC core, divided into two at the stator, and then returns to the SMC core to create a closed path. The equivalent magnetic circuit is represented in Fig. 3(b) and flux linkage calculated as follows: 


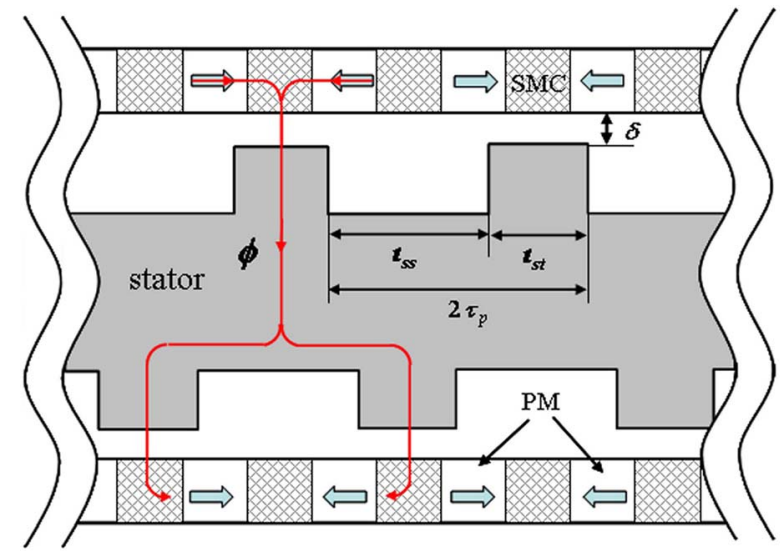

(a)

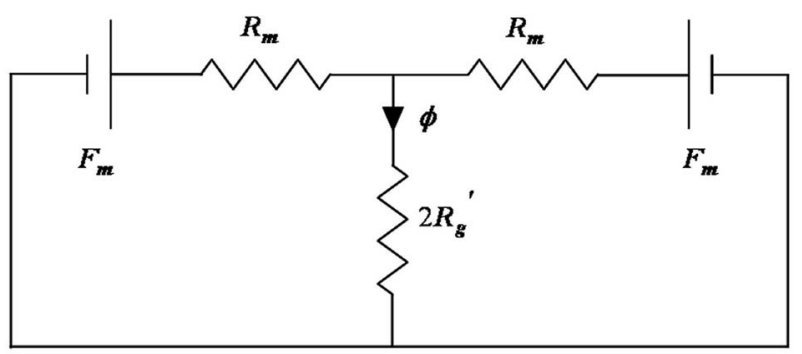

(b)

Fig. 3. Equivalent magnetic circuit of TFLM: simplified magnetic flux flow (b) equivalent circuit.

$$
\begin{gathered}
\phi_{\min }=\frac{B_{r} l_{m}}{\mu_{m}\left(\frac{2 \delta^{\prime}}{A_{g}}+\frac{l_{m}}{2 \mu_{m} A_{m}}\right)} \\
\left(\delta^{\prime}=\delta \times K_{C}\right)
\end{gathered}
$$

where, $B_{r}, \mu_{m}, A_{m}, A_{g}, \delta^{\prime}$ are the residual flux density, relative permeability of permanent magnet, magnet area in the direction of magnetization, area of the air gap region, and effective air gap length, taking Carter's coefficients $\left(K_{C}\right)$ for the stator tooth opening into the account, respectively [4].

With the information of the average magnetic flux density derived from the average thrust, the maximum flux linkage can be calculated at full-load condition as follows:

$$
\phi_{\max }=\frac{\sqrt{2 \mu_{0} \times F_{x d}} \times(\text { force generating area } / 2)}{(2 / \pi)}
$$

\subsubsection{Number of turns}

By neglecting the resistance voltage drop and leakage flux, the number of turns $(N)$ of each phase winding should satisfy the following equation. When the mover runs at the rated speed $\left(v_{\text {rated }}\right)$, voltage drop due to the flux linkage variation should be less than the input voltage $\left(V_{\text {input }}\right)$ :

$$
\begin{aligned}
N \frac{d \phi}{d t} & =N \frac{\Delta \phi}{\Delta t} \\
& =N \frac{\phi_{\max }-\phi_{\min }}{\frac{0.5 \tau_{p}}{v_{\text {rated }}}} \cdot \frac{\pi}{2}<V_{\text {input }}
\end{aligned}
$$

\subsubsection{Applied MMF}

The applied MMF $(F)$ has a relation with the important design parameters, including the thrust density. It is also influenced by the properties of the used magnetic materials due to the saturation effect of the magnetic flux density. The following shows the empirical relations of the MMF with other design variables. When both the TFLM mover and stator are made of silicon steel, (7) can be adapted. When one part of the TFLM is replaced with a soft magnetic composite (SMC) material having a much lower saturation level than that of silicon steel, the MMF has the relation with thrust density as (8). The constant (c) is the index representing the saturation level of the magnetic flux density in the magnetic circuit. When $c$ is zero, there is a linear relation between thrust density and applied MMF. When $c$ is one, the thrust density is heavily saturated with the applied MMF [5]. In the general case, the constant $c$ is determined by the value between 0.1 and 0.4 , at first by considering the thrust density at the rated MMF; it provides reasonable results in the initial design and parametric study:

$$
\begin{gathered}
F=\frac{\tau_{p}+10}{25} \cdot \frac{\delta+1}{2}\left[\frac{14\left(1+0.2 \frac{\tau_{p}}{w_{s t}}\right) \cdot F_{x d} \times 2+}{\frac{c}{10^{3}}\left(1+0.2 \frac{\tau_{p}}{w_{s t}}\right)^{3} \cdot\left(F_{x d} \times 2\right)^{3}}\right] \\
F=\frac{\tau_{p}+10}{25} \cdot \frac{\delta+1}{2}\left[\frac{34.5\left(1+0.2 \frac{\tau_{p}}{w_{s t}}\right) \cdot F_{x d}+}{\frac{c}{10^{3}}\left(1+0.2 \frac{\tau_{p}}{w_{s t}}\right)^{3} \cdot\left(F_{x d} \times 3\right)^{3}}\right]
\end{gathered}
$$

\subsubsection{Coil size}

The rated current can be determined with the number of turns from (6) and the applied MMF from (7) or (8). When the current density $(J)$ is predetermined by considering the cooling conditions of the motor, the diameter of the bare conductor $\left(d_{W}\right)$ can be determined as follows:

$$
d_{W}=2 \times \sqrt{\frac{F}{N} \cdot \frac{1}{J \pi}}
$$

In calculating the winding area, wire insulation and fill factor should be considered. With these procedures, a three- 
Table 1. Specifications of the designed motor

\begin{tabular}{lll}
\hline \hline Symbol & Quantity \\
\hline DC link voltage (V) & 540 \\
Number of turns & $46 \times 2$ (parallel) \\
Rated MMF (AT) & 1,900 \\
Rated speed (m/s) & 3 \\
Air gap length (mm) & 1.0 \\
Permanent magnet & NdFeB $\quad \mathrm{Br}=1.2 \mathrm{~T}$ \\
\hline \multirow{4}{*}{$\tau_{p}(\mathrm{~mm})$} & 10 \\
& $t_{s t}(\mathrm{~mm})$ & 7 \\
Stator & $w_{s t}(\mathrm{~mm})$ & 78 \\
& $h_{s t}(\mathrm{~mm})$ & 65 \\
& Length $(\mathrm{mm})$ & 8,000 \\
& material & $\mathrm{S} 18$ \\
\hline \multirow{4}{*}{ Mover } & $t_{p m}$ & 5 \\
& $w_{c}$ & 18 \\
& $h_{c}$ & 20 \\
& Length $(\mathrm{mm})$ & 215 (PM:21, SMC:22) \\
\hline
\end{tabular}

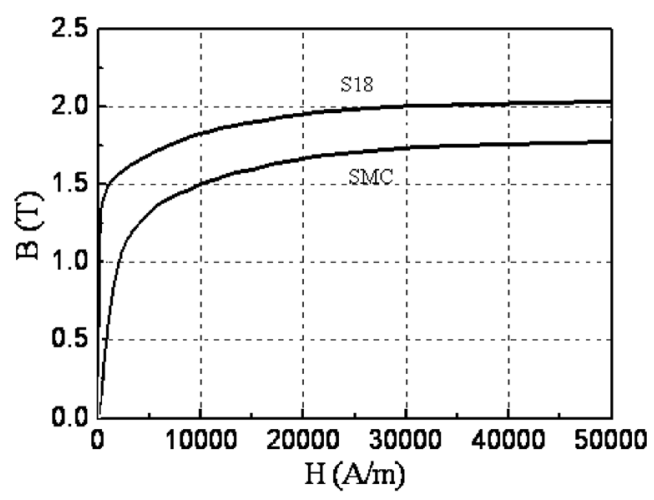

Fig. 4. B-H curves of the magnetic materials.

phase TFLM having $1,000 \mathrm{~N}$ thrust can be designed. The important design specifications are listed in Table 1 and the B-H curves of the magnetic materials shown in Fig. 4.

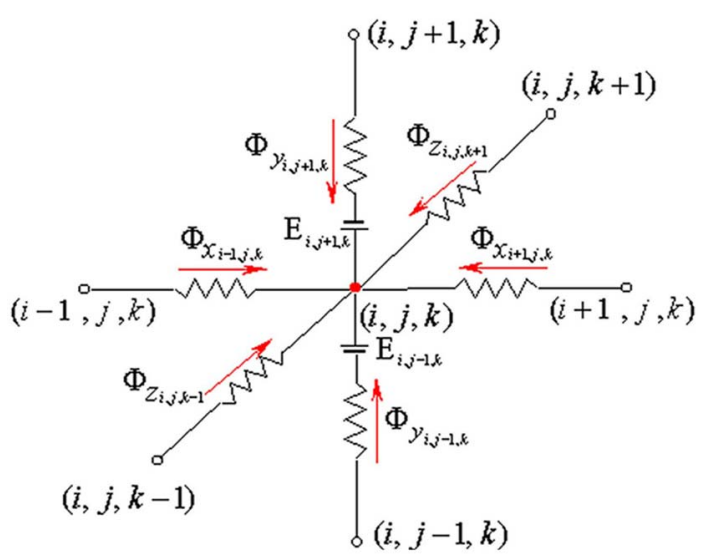

Fig. 5. Conceptual model of the EMCN method at a node.

\subsection{Basic design verification by EMCN}

Three-dimensional numerical analysis by an EMCN method was introduced to verify the proposed design procedures of the TFLM. The EMCN method represents the analysis region with the connection of permeance elements and is based on the magnetic flux continuity at each node. The conceptual model of the equivalent magnetic circuit at a node is given in Fig. 5. The sum of magnetic flux flowing into that node is equal to the sum of the magnetic flux flowing out of that node. This can be stated as follows by considering a total of six branches with magnetic flux flowing in each direction:

$$
\begin{aligned}
\Sigma \Phi_{\text {node }}= & \Phi_{x, i-1, j, k}+\Phi_{x, i+1, j, k}+\Phi_{y, i, j-1, k}+ \\
& \Phi_{y, i, j+1, k}+\Phi_{z, i, j, k-1}+\Phi_{z, i, j, k+1}
\end{aligned}
$$

Each magnetic flux in (10) can be calculated with permeance between nodes, magnetic scalar potential at each node, and equivalent MMF by external sources; finally, this gives the system matrix (11):

$$
[P]\{U\}=\{F\}
$$

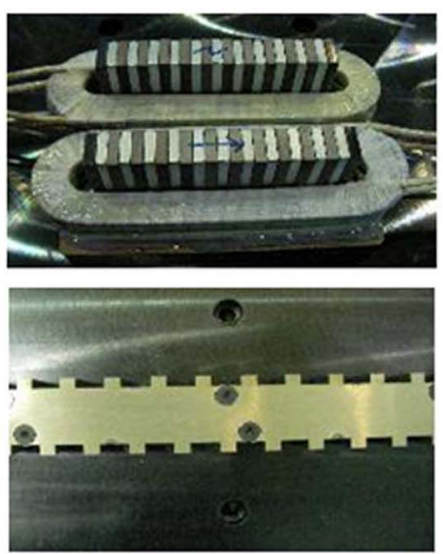

(a)

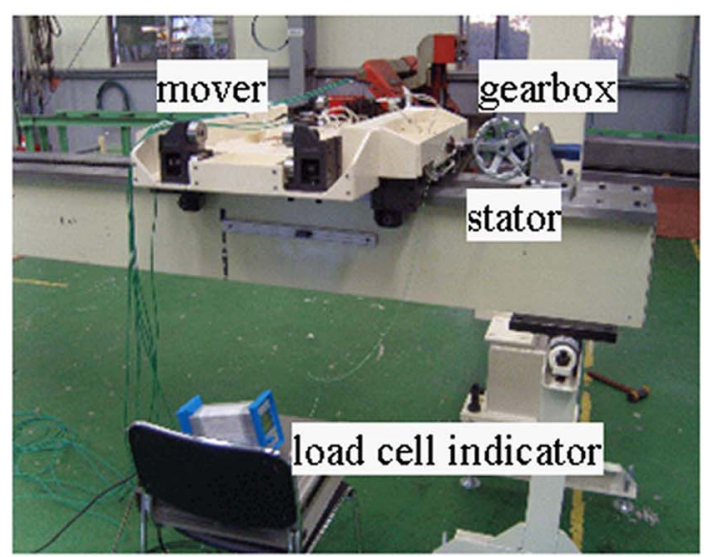

(b)

Fig. 6. Experiment setup: (a) prototyped mover and stator (b) thrust measure. 
where $[P]$ is the permeance coefficient matrix, $\{U\}$ the vector of magnetic scalar potential, and $\{F\}$ the forcing vector [6].

\section{Experimental Results}

To validate the proposed design procedure, the mover and stator of the $1,000 \mathrm{~N}$-class of the TFLM is manufactured as shown in Fig. 6(a), and the thrust measured at the right place of installation of the TFLM as shown in Fig. 6(b). Fig. 7 compares the thrust densities obtained by different methods. In the proposed design method, the index $(c)$ representing the saturation level of the thrust determines the shape of the graph. When $c$ is near 0.15 , it is very similar to the results of the experiment and threedimensional EMCN method. At the rated MMF, the thrust density is approximately $50 \mathrm{kN} / \mathrm{m}^{2}$. Due to the lower saturation level of SMC, it has a somewhat low thrust density.

In Fig. 8, the thrust profiles calculated by the three-

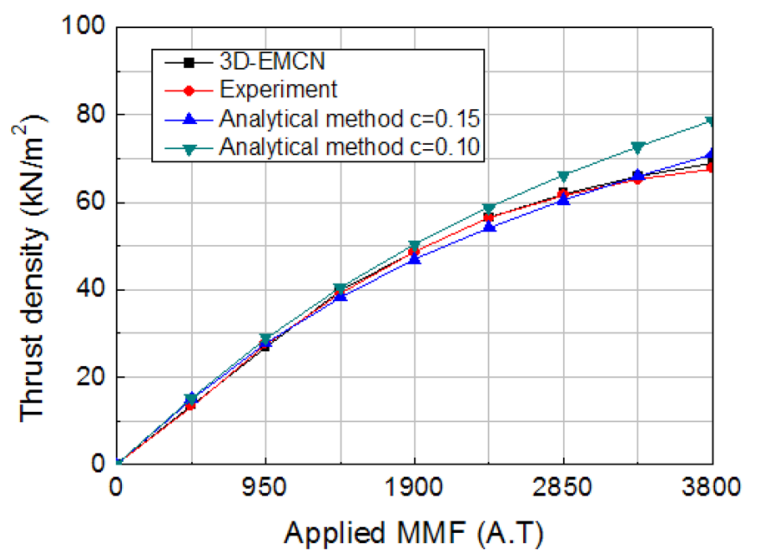

Fig. 7. Comparison of thrust obtained by different method with the variation of applied MMF.

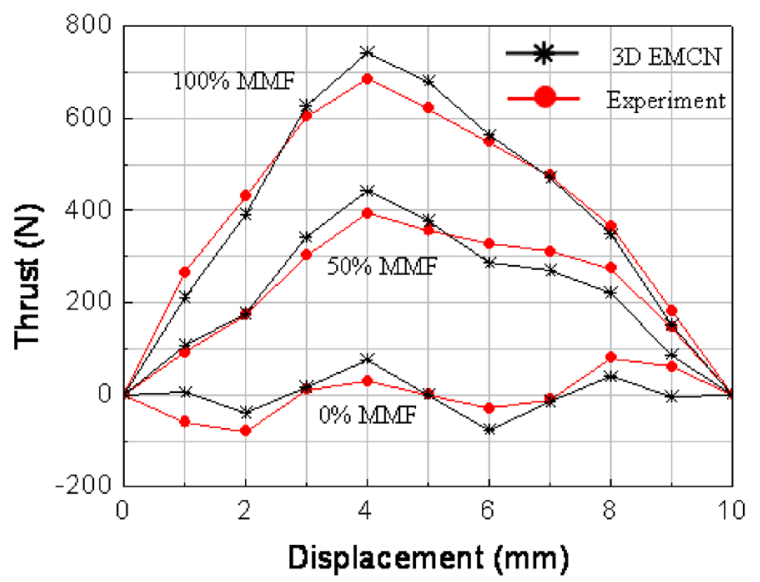

Fig. 8. Comparison of thrust profiles with the variations of applied MMF. dimensional EMCN method are compared with the experimental results with the variations of applied MMF. At the rated MMF, the average thrust value per phase is 379 and $381 \mathrm{~N}$ by the experiment and three-dimensional EMCN method, respectively. Fig. 9 shows the results of a dynamic experiment. The total moving mass was $650 \mathrm{~kg}$ and it is accelerated with an average acceleration of $1.6 \mathrm{~m} / \mathrm{s}^{2}$, indicating that the TFLM produces $1,000 \mathrm{~N}$ thrust, the rated force of the designed motor. In a corresponding current profile, at low speed, the phase current also has the value of the rated current. This shows the designed motor produces rated force at the rated current in the dynamic situation. In the acceleration region, the input current increases with the speed due to the increase in electromechanical losses. Nonetheless, in the deceleration region, the input current is much less due to the mechanical friction in opposite direction with the motion of the moving object. By these static and dynamic experiments, it was verified that the prototyped machine manufactured by the proposed design method satisfies the design specifications, showing the possibility of the proposed method to be applicable to the TFLM design.

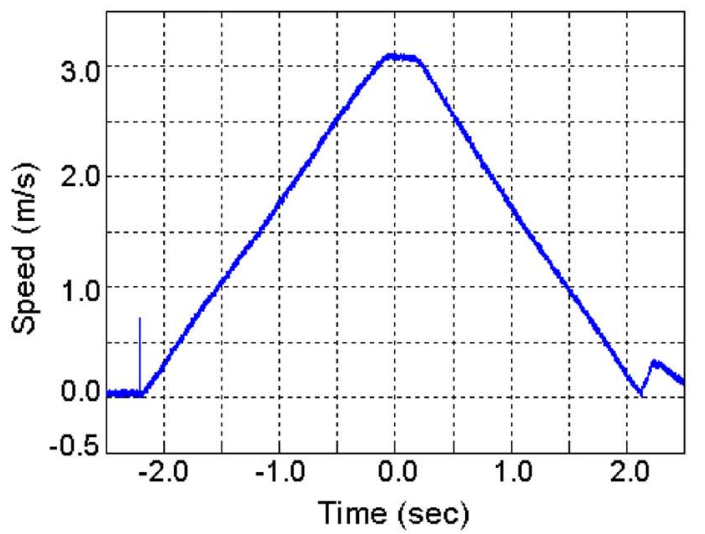

(a)

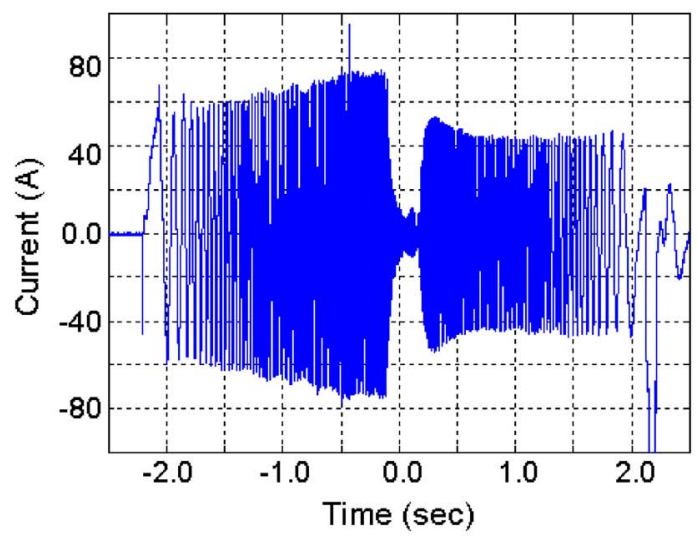

(b)

Fig. 9. Dynamic experiment results: (a) speed profile (b) current profile. 


\section{Conclusions}

This paper proposes a design procedure of a TFLM. Important design variables, including basic dimensions, numbers of turns, applied MMF, and thrust density are determined based on analytical method and empirical formula. The usefulness of the proposed design procedure is verified by a three-dimensional EMCN method and the experimental results of a prototyped machine, showing good agreement with each other and satisfying the expected performance on the thrust and speed. The proposed design procedures could be adapted to the design of other kinds of transverse flux machines, including a rotating type transverse flux motor.

\section{References}

[1] Junghwan Chang, Jiwon Kim, Dohyun Kang, and Deokje Bang, J. Magnetics 15, 64 (2010).

[2] Junghwan Chang, Dohyun Kang, Jiyoung Lee, and Jungpyo Hong, IEEE Trans. Magn. 41, 1936 (2005).

[3] D. H. Kang, Y. H. Chun, and H. Weh, IEE Proc.-Electr. Power Appl. 150, 493 (2003).

[4] Duane Hanselman, Brushless Permanent Magnet Motor Design, Magna Physics Pub., Lebanon, OH (2003).

[5] Hervert Weh, KERI-report on the Design of Transverse Flux Machine (2006).

[6] V. Ostovic, Dynamics of Saturated Electric Machine, Springer-Verlag, New York, NY (1989). 